Entanglement dynamics in superconducting qubits affected by local bistable impurities

This article has been downloaded from IOPscience. Please scroll down to see the full text article.

2012 Phys. Scr. 2012014019

(http://iopscience.iop.org/1402-4896/2012/T147/014019)

View the table of contents for this issue, or go to the journal homepage for more

Download details:

IP Address: 147.163.21.217

The article was downloaded on 22/02/2012 at 14:20

Please note that terms and conditions apply. 


\title{
Entanglement dynamics in superconducting qubits affected by local bistable impurities
}

\author{
R Lo Franco ${ }^{1,3}$, A D’Arrigo ${ }^{2}$, G Falci $^{2}$, G Compagno $^{3}$ and E Paladino ${ }^{2}$ \\ ${ }^{1}$ Centro Siciliano di Fisica Nucleare e Struttura della Materia (CSFNSM) and Dipartimento di Fisica e \\ Astronomia, Università di Catania, via Santa Sofia 64, 95123 Catania, Italy \\ ${ }^{2}$ Dipartimento di Fisica e Astronomia, Università di Catania, and CNR-IMM Matis, via Santa Sofia 64, \\ 95123 Catania, Italy \\ ${ }^{3}$ CNISM and Dipartimento di Fisica, Università di Palermo, via Archirafi 36, 90123 Palermo, Italy \\ E-mail: lofranco@ fisica.unipa.it
}

Received 16 September 2011

Accepted for publication 29 September 2011

Published 17 February 2012

Online at stacks.iop.org/PhysScr/T147/014019

\begin{abstract}
We study the entanglement dynamics for two independent superconducting qubits, each affected by a bistable impurity generating random telegraph noise (RTN) at pure dephasing. The relevant parameter is the ratio $g$ between the qubit-RTN coupling strength and the RTN switching rate, which captures the physics of the crossover between Markovian and non-Markovian features of the dynamics. For identical qubit-RTN subsystems, a threshold value $g_{\text {th }}$ of the crossover parameter separates exponential decay and the onset of revivals; different qualitative behaviors also show up by changing the initial conditions of the RTN. We also show that, for different qubit-RTN subsystems, when both qubits are very strongly coupled to the RTN, an increase of entanglement revival amplitude may occur during the dynamics.
\end{abstract}

PACS numbers: 03.67.Mn, 03.65.Yz, 03.67.-a

(Some figures may appear in colour only in the online journal)

\section{Introduction}

Entanglement dynamics has been intensively studied for bipartite quantum systems interacting with quantum environments (independent or common). It presents, depending on the Markovian or non-Markovian nature of the environments, phenomena such as entanglement sudden death (ESD) [1], revivals [2-4] or trapping [5, 6]. Entanglement dynamics has also been analyzed in some solid-state systems, such as superconducting qubits or quantum dots, which are promising candidates for realizing quantum information processing [7-10]. Considerable progress has been made over the last decade toward the implementation of a controlled solid-state quantum computer. In particular, superconducting high-fidelity single-qubit gates with coherence times of $\sim 1 \mu$ s are currently available [11, 12]. High-fidelity Bell states of superconducting qubits have also been prepared $[13,14]$. Superconducting nanodevices are usually affected by broadband noise, with typical power spectra displaying a $1 / f$ low-frequency behavior followed by a white or ohmic flank [15-18]. The dynamics of entanglement in these devices has recently been analyzed distinguishing the effects of adiabatic and quantum noise [9]. The disentanglement process has also been studied by the quasi-Hamiltonian method when two independent superconducting qubits are locally affected by random telegraph noise (RTN) [19].

In this paper we consider a model, relevant to the solid-state system [17], consisting of two non-interacting qubits, each affected by a single bistable impurity inducing RTN at the pure-dephasing working point. In addition, this model can be exactly solvable in analytic form and captures the physics of the crossover between a Markovian and a non-Markovian environment under quite general conditions. The relevant parameter separating the two regimes is the ratio between the qubit-RTN coupling constant and the switching rate of the impurity. We analyze the entanglement 
dynamics for both identical and different conditions of the two local qubit-RTN. In section 2 we introduce the relevant single qubit-RTN model, while in section 3 we describe the entanglement dynamics under various initial noise conditions. In section 4, we present our concluding remarks.

\section{Model: a qubit under pure-dephasing random telegraph noise}

Our system consists of a couple of independent superconducting qubits, $A$ and $B$, each affected by a bistable impurity generating RTN at pure dephasing. The total Hamiltonian is $H_{\mathrm{tot}}=H_{A}+H_{B}$, where for each qubit the Hamiltonian at pure dephasing is $(\hbar=1)$ [17]

$$
H=-(\Omega / 2) \sigma_{z}-(v / 2) \xi(t) \sigma_{z},
$$

where $\xi(t)$ is a stochastic process producing RTN switching at a rate $\gamma$ between \pm 1 , and $v$ is the qubit-RTN coupling constant. The power spectrum of the unperturbed equilibrium fluctuations of $\xi(t)$ is $s(\omega)=v^{2} \gamma /\left[2\left(\gamma^{2}+\omega^{2}\right)\right]$. The relevant parameter is the ratio $g=v / \gamma$ that permits us to analyze the crossover between a Markovian environment for weakly coupled impurities $(g<1)$ and a non-Markovian environment for strong coupled impurities $(g>1)$ [17]. The exact evolution of single-qubit coherence $q(t) \equiv \rho_{01}(t) / \rho_{01}(0)$ has been found in [17] and is given by

$$
q(t)=\mathrm{e}^{-\mathrm{i}(\Omega+v / 2) t}\left[A \mathrm{e}^{-\frac{\gamma(1-\alpha) t}{2}}+(1-A) \mathrm{e}^{-\frac{\gamma(1+\alpha) t}{2}}\right],
$$

where $A=\frac{1}{2 \alpha}\left(1+\alpha-\mathrm{i} g \delta p_{0}\right)$ and $\alpha=\sqrt{1-g^{2}}$. Note that $\delta p_{0}$ is a degree of freedom depending on the initial conditions of the RTN and is not present in previous analysis of entanglement dynamics under RTN [19]. In particular, $\delta p_{0}$ can take the value $\delta p_{0}=0$ (corresponding to the value of thermodynamical equilibrium) or the values $\delta p_{0}= \pm 1$. The form of equation (1) clearly shows the different roles of weakly and strongly coupled impurities in the decoherence process. Dephasing comes from the sum of two exponential terms. If $g \ll 1$ only the first of these terms is important and the corresponding rate is $\approx v^{2} /(4 \gamma)$, coinciding with the golden rule; if $g \gg 1$ the two terms are of the same order and the decay rate is $\sim \gamma$ [17].

\section{Dynamics of entanglement}

The two-qubit density matrix elements are evaluated in the computational basis $\mathcal{B}=\{|0\rangle \equiv|00\rangle,|1\rangle \equiv|01\rangle,|2\rangle \equiv|10\rangle$, $|3\rangle \equiv|11\rangle\}$, where $H_{i}\left|0_{i}\right\rangle=-\frac{\Omega_{i}}{2}\left|0_{i}\right\rangle, \quad H_{i}\left|1_{i}\right\rangle=\frac{\Omega_{i}}{2}\left|1_{i}\right\rangle \quad(i=$ $A, B)$, where $H_{i}=-(\Omega / 2) \sigma_{z}$. We consider as initial states the extended Werner-like (EWL) states expressed by the density matrices [20]

$$
\hat{\rho}_{1}=r\left|1_{a}\right\rangle\left\langle 1_{a}\left|+\frac{1-r}{4} \mathbb{1}_{4}, \quad \hat{\rho}_{2}=r\right| 2_{a}\right\rangle\left\langle 2_{a}\right|+\frac{1-r}{4} \mathbb{1}_{4}
$$

whose pure parts are the one and two-excitation Bell-like states $\left|1_{a}\right\rangle=a|01\rangle+b|10\rangle,\left|2_{a}\right\rangle=a|00\rangle+b|11\rangle$, where the subscript $a$ identifies the initial degree of entanglement of the pure part and $|a|^{2}+|b|^{2}=1$. The density matrix of EWL states is non-vanishing only along the diagonal and anti-diagonal ( $\mathrm{X}$ form) [20], and this structure is maintained at $t>0$ in the system we are considering (pure dephasing). Using the concurrence [21] $C$ to quantify entanglement, the initial entanglement is equal for both the EWL states of equation (2) and reads $C_{\rho_{1}}(0)=C_{\rho_{2}}(0)=2 \max \{0,(|a b|+$ $1 / 4) r-1 / 4\}$. Initial states are thus entangled for $r>r^{*}=$ $(1+4|a b|)^{-1}$. Moreover, the purity $P=\operatorname{Tr}\left(\rho^{2}\right)$ of EWL states is $P=\left(1+3 r^{2}\right) / 4$. Entangled states with purity $\approx 0.87$ and fidelity to ideal Bell states $\approx 0.90$ have been experimentally generated [14]: these states may be approximately described as EWL states with $r_{\exp } \approx 0.91$.

In order to obtain the concurrence at time $t$, we need the evolved two-qubit density matrix that can be evaluated by the knowledge of the single-qubit density matrix evolution, according to a standard procedure [2]. The initial EWL states, during the pure-dephasing evolution, maintain the diagonal elements unchanged and the anti-diagonal elements depend on the product of single-qubit coherences. The concurrences at time $t$ for the two initial states of equation (2) are given by, respectively, $C_{\rho_{1}}(t)=2 \max \left\{0, K_{1}(t)\right\}$ and $C_{\rho_{2}}(t)=$ $2 \max \left\{0, K_{2}(t)\right\}$, where $K_{1}(t)=\left|\rho_{12}(t)\right|-\sqrt{\rho_{00}(t) \rho_{33}(t)}$, $K_{2}(t)=\left|\rho_{03}(t)\right|-\sqrt{\rho_{11}(t) \rho_{22}(t)}$. In our case, we have $K_{1}(t)=K_{2}(t)=K(t)$, so that the two concurrences are equal for both initial states $C_{\rho_{1}}(t)=C_{\rho_{2}}(t)=C(t)$. In the following, we consider identical qubits $\left(\Omega_{A}=\Omega_{B}=\Omega\right)$ and we distinguish the cases of equal RTNs $g_{A}=g_{B}=g$ and of different RTNs $g_{A} \neq g_{B}$ for the two subsystems.

\subsection{Identical random telegraph noises}

Consider the case $g_{A}=g_{B}=g$ and equal initial conditions. We obtain $C(t)=\max \{0,2 K(t)\}$ with

$$
K(t)=r|a| \sqrt{1-|a|^{2}}|q(t)|^{2}-(1-r) / 4,
$$

where $q(t)$ is the single-qubit coherence of equation (1). We now investigate how the concurrence depends on different initial conditions of the system and state parameters. Equation (3) clearly shows the role of the purity parameter $r$ in concurrence evolution: for pure states, $r=1$, there cannot be an ESD and the entanglement goes asymptotically to zero similarly to single-qubit coherence; on the other hand, any value of $r$ inside the interval $r^{*}<r<1$ determines a threshold value for the first term of equation (3) to be overcome in order to have non-zero entanglement (of course, $0<|a|<1$ ). The dynamics of entanglement is shown in figure 1 when $g=0.5,5$ and $\delta p_{0}=$ $0, \pm 1$, for initial states with $r=r_{\exp }=0.91$ and $a=b=$ $1 / \sqrt{2}$ whose initial concurrence is $C(0)=0.865$. Similarly to other works [2, 19], for weak coupling $(g<1$, Markovian environment) there is a simple (exponential) decay, while for strong coupling ( $g>1$, non-Markovian environment) there are damped revivals after dark periods of entanglement (see figure 1). It is, however, worth noting that, whereas for a single qubit the crossover between Markovian (exponential) and non-Markovian (oscillating) decay is identified by $g=1$, for the two-qubit system, depending on initial state parameters, a threshold value $g_{\text {th }}>1$ exists separating exponential decay and the onset of revivals. This $g_{\text {th }}$ can be found analytically [22] and its dependence on $r$ (for $a=b=1 / \sqrt{2}$ ) is displayed in figure 1(b) (for 

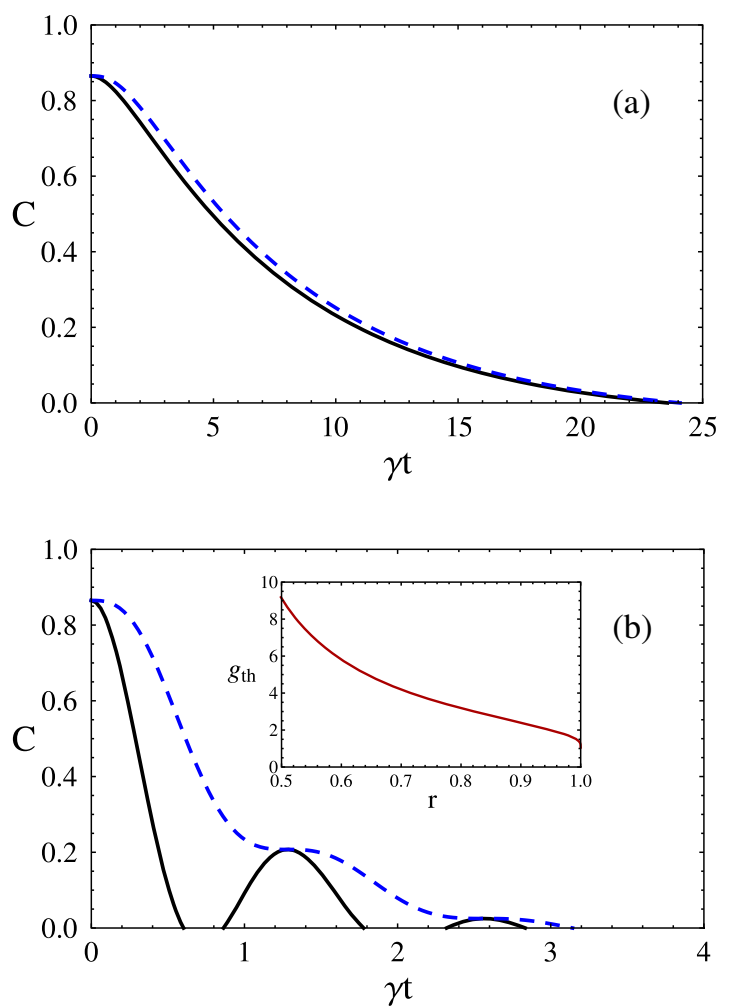

Figure 1. Concurrences versus the dimensionless time $\gamma t$ with $r=r_{\text {exp }}=0.91$ and $a=b=1 / \sqrt{2}$ for $g=0.5$ (a) and $g=5$ (b). Solid black lines are for $\delta p_{0}=0$, while dashed blue lines are for $\delta p_{0}= \pm 1$. The inset of panel (b) displays $g_{\text {th }}$ versus $r$ for $a=b=1 / \sqrt{2}$.

$r=1$ it is $\left.g_{\text {th }}=1\right)$. When $r=1$ the entanglement goes to zero asymptotically (oscillating and vanishing at given times for $g>g_{\text {th }}$ ), analogous to the results already found for the case of adiabatic (low-frequency) noise due to collective impurities generating $1 / f$ noise [9]. In contrast, when $r<1$ there is always ESD for $g \leqslant g_{\text {th }}$ and a 'final death' (after revivals) for $g>g_{\text {th }}$, where by final death we mean the definitive disappearance of entanglement. Different RTN initial conditions (values of $\delta p_{0}$ ) qualitatively affect the entanglement dynamics for $g>g_{\text {th }}$ (strong memory effects), while they leave it practically unchanged for $g \leqslant g_{\text {th }}$ (weak memory effects), as shown in figure 1. In particular, when $g>g_{\text {th }}$, the concurrence for $\delta p_{0}= \pm 1$ is always larger than that for $\delta p_{0}=0$ with small beats touching the peaks of the revivals appearing in the corresponding curve for $\delta p_{0}=0$; the final death time is also longer than the previous one (see figure 1).

In the limit of small $g$ (Markovian noise) and for $\delta p_{0}=0$, only the first term is important in equation (1) and, from equation (3), we may estimate the ESD times (giving, however, a good match with plots up to $g=0.9$ )

$$
\gamma t_{\mathrm{ESD}}=-\frac{2}{1-\sqrt{1-g^{2}}} \ln \left(\frac{\sqrt{\left(1-g^{2}\right)(1-r) / r|a| \sqrt{1-|a|^{2}}}}{1+\sqrt{1-g^{2}}}\right) .
$$

These ESD times are plotted in figure 2 as a function of $g$ for three different values of $r$. They decrease as $g$ increases, with a reduction of about an order of magnitude going from $g=0.1$ to $g=0.4$.

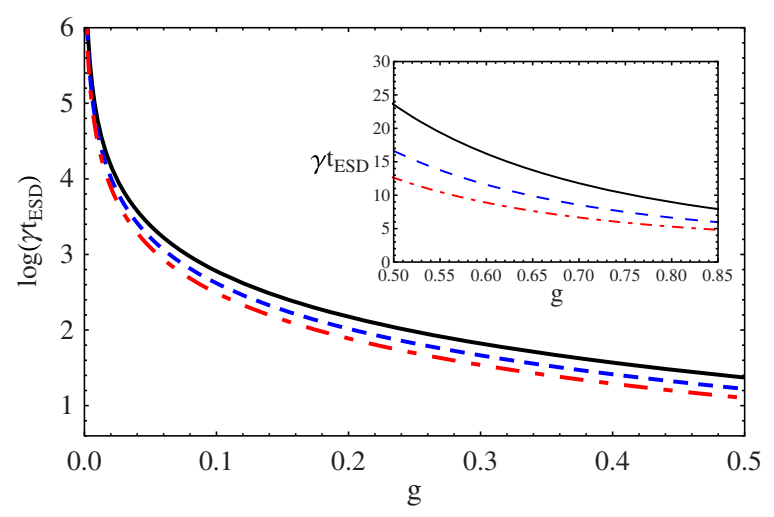

Figure 2. ESD times $t_{\mathrm{ESD}}$ (scaled with $\gamma$ ) versus $g$, with $a=b=1 / \sqrt{2}$ and $\delta p_{0}=0$, for $r_{\exp }=0.91$ (black solid line), $r=0.8$ (blue dashed line) and $r=0.7$ (red dot-dashed line). The inset shows the range $g>0.5$.

\subsection{Different random telegraph noises}

We now consider the more realistic case of qubits affected by different RTNs $g_{A} \neq g_{B}$, with $\delta p_{0}=0$ for both. The function $K(t)$ is now given by equation (3) replacing $|q(t)|^{2}$ with $\left|q_{A}(t) q_{B}(t)\right|$, where $q_{i}(t)(i=A, B)$ is the coherence of qubit $i$ given in equation (1). The entanglement dynamics for different values of $g_{A}, g_{B}$ is displayed in figure 3 . We assume the rate $\gamma$ to be fixed for both impurities and different values of $v_{A}, v_{B}$. When a qubit, for instance qubit $B$, is not affected by RTN $\left(g_{B}=0\right)$, we find a qualitative behavior analogous to that observed for identical RTNs, with a threshold value $g_{\text {Ath }}>1$ after which revivals occur. On the other hand, when one of the two qubits is affected by a strong non-Markovian RTN (for instance, $g_{B}=2$ ), as $g_{A}$ increases the revivals tend to disappear after a certain $g_{A}>1$ (indeed, when $g_{A}=g_{B}=2$ the value of $g_{\text {th }}$ is larger than 2, as seen in the previous section) and then reappear for larger values of $g_{A}$ with final death times shorter and shorter. With further increasing the fixed value of $g_{B}$, other new qualitative behaviors appear when $g_{A}$ increases in the strong coupling domain. The inset of figure 3(b) shows the presence of entanglement revivals whose amplitude increases with respect to the previous one for the values $g_{A}=10, g_{B}=5$. Similar behaviors occur for initial pure states $(r=1)$ but without dark periods. These behaviors are due to the different contributions of the two single-qubit dynamics.

\section{Conclusions}

In this paper, we have analyzed the entanglement dynamics in a system of two initially entangled independent superconducting qubits ( $A$ and $B$ ), each affected by impurity-induced RTN at pure dephasing. A crucial role in determining the behavior of entanglement dynamics is played by the ratio between qubit-RTN coupling $v$ and switching rate $\gamma, g=v / \gamma$. For identical RTNs, we found that, in spite of the fact that for the single-qubit dynamics the crossover between Markovian and non-Markovian behaviors is identified by $g=1$, for an initially entangled two-qubit system this threshold is, in general, shifted to a value $g_{\text {th }}>1$ depending on the initial state (for initial pure states $g_{\text {th }}=1$ ). In the case when the RTN has the initial value $\delta p_{0}=0$ 

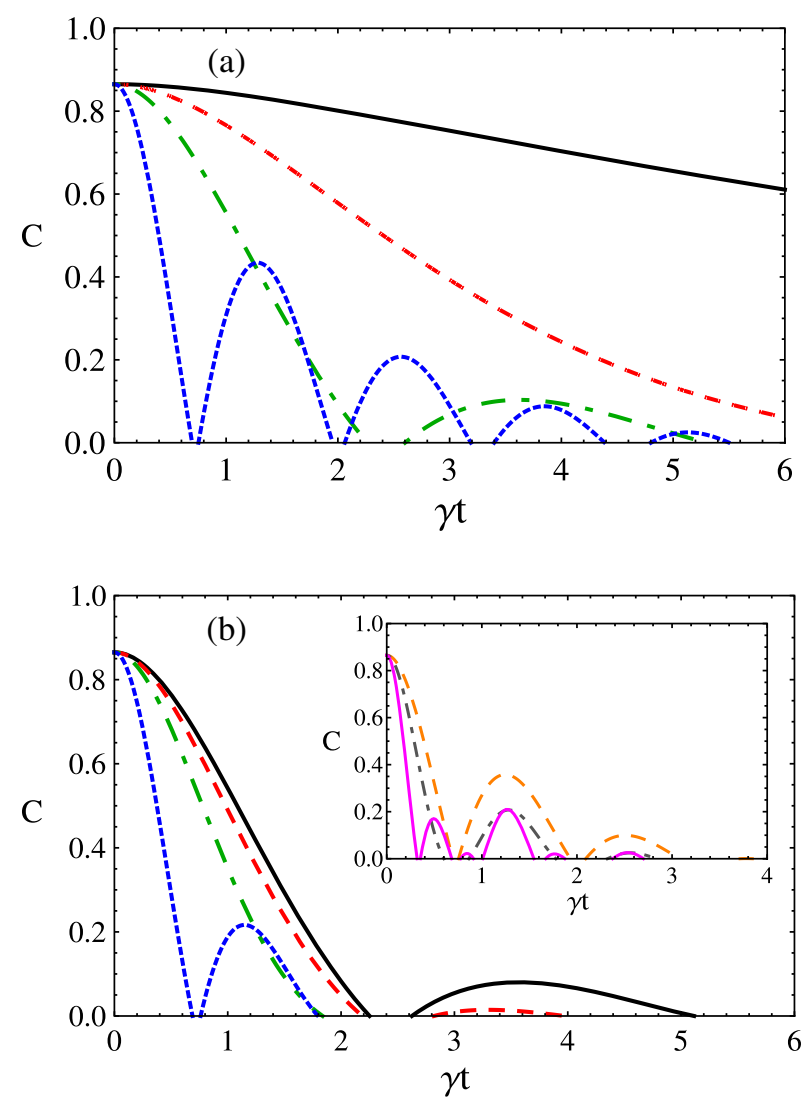

Figure 3. Concurrences versus $\gamma t$ for $g_{B}=0$ (a) and $g_{B}=2$ (b) and values of $g_{A}$ equal to 0.5 (solid black line), 1.1 (red dashed line), 2 (green dot-dashed line) and 5 (blue dotted line). In the inset of panel (b), we set $g_{B}=5$ with values of $g_{A}$ equal to 1.1 (orange dashed line), 5 (gray dot-dashed line) and 10 (magenta solid line). Initial conditions: $r=r_{\exp }=0.91, a=b=1 / \sqrt{2}, \delta p_{0}=0$.

(thermodynamical equilibrium), we have retrieved the known behaviors of exponential (Markovian) decay for $g \leqslant g_{\text {th }}$ and of oscillating (non-Markovian) decay with revivals for $g>g_{\text {th }}$. We then found new qualitative behaviors, in the non-Markovian regime $\left(g>g_{\text {th }}\right.$, relevant memory effects), by changing the initial value of RTN, $\delta p_{0}$. For example, if $\delta p_{0}= \pm 1$, the entanglement never vanishes at intermediate times and no revivals occur, with a final death time longer than that for $\delta p_{0}=0$. We finally considered the case when the two local qubit-RTN conditions differ, in particular $g_{A} \neq g_{B}$. Entanglement dynamics may behave quite differently from that for identical subsystems. In particular, when both $g_{A}$,
$g_{B}$ are in the strong non-Markovian domain, a new feature in the entanglement dynamics shows up; that is, there can be entanglement revivals whose amplitude increases with respect to the previous one.

The simple model, relevant to solid-state systems, analytically studied here displays the richness of behaviors of entanglement dynamics and its crucial dependence on both system-environment parameters and initial conditions.

\section{Acknowledgment}

This work was partially supported by the EU through grant no. PITN-GA-2009-234970.

\section{References}

[1] Yu T and Eberly J H 2004 Phys. Rev. Lett. 93140404

[2] Bellomo B, Lo Franco R and Compagno G 2007 Phys. Rev. Lett. 99160502

[3] Mazzola L, Maniscalco S, Piilo J, Suominen K A and Garraway B M 2009 Phys. Rev. A 79042302

[4] López C E, Romero G and Retamal J C 2010 Phys. Rev. A 81062114

[5] Bellomo B, Lo Franco R, Maniscalco S and Compagno G 2008 Phys. Rev. A 78060302

[6] Bellomo B, Lo Franco R, Maniscalco S and Compagno G 2010 Phys. Scr. T140 014014

[7] Liu Y X, Özdemir S K, Koashi M and Imoto N 2002 Phys. Rev. $\mathbf{6 5} 042326$

[8] Tsomokos D I, Hartmann M J, Huelga S F and Plenio M B 2007 New J. Phys. 979

[9] Bellomo B, Compagno G, D'Arrigo A, Falci G, Lo Franco R and Paladino E 2010 Phys. Rev. A 81062309

[10] Bellomo B, Compagno G, Lo Franco R, Ridolfo A and Savasta S 2011 Phys. Scr. T143 014004

[11] Vion D et al 2002 Science 296886

[12] Schreier J A et al 2008 Phys. Rev. B 77180502

[13] Steffen M et al 2006 Science 3131423

[14] DiCarlo L et al 2009 Nature 460240

[15] Ithier G et al 2005 Phys. Rev. B 72134519

[16] Astafiev O et al 2004 Phys. Rev. Lett. 93267007

[17] Paladino E, Faoro L, Falci G and Fazio R 2002 Phys. Rev. Lett. 88228304

[18] Falci G, D'Arrigo A, Mastellone A and Paladino E 2005 Phys. Rev. Lett. 94167002

[19] Zhou D, Lang A and Joynt R 2010 Quantum Inf. Process. 9727

[20] Bellomo B, Lo Franco R and Compagno G 2008 Phys. Rev. A 77032342

[21] Wootters W K 1998 Phys. Rev. Lett. 802245

[22] Lo Franco R et al 2012 in preparation 